\title{
Modeling Implantable Passive Mechanisms for Modifying the Transmission of Forces and Movements Between Muscle and Tendons
}

\author{
Taymaz Homayouni*, Kelsey N. Underwood, Kamin C. Beyer, Elon R. Martin, Christopher H. Allan, \\ and Ravi Balasubramanian
}

\begin{abstract}
This paper explores the development of biomechanical models for evaluating a new class of passive mechanical implants for orthopedic surgery. The proposed implants take the form of passive engineered mechanisms, and will be used to improve the functional attachment of muscles to tendons and bone by modifying the transmission of forces and movement inside the body. Specifically, we present how two types of implantable mechanisms may be modeled in the open-source biomechanical software OpenSim. The first implant, which is proposed for hand tendon-transfer surgery, differentially distributes the forces and movement from one muscle across multiple tendons. The second implant, which is proposed for knee-replacement surgery, scales up the forces applied to the knee joint by the quadriceps muscle. This paper's key innovation is that such mechanisms have never been considered before in biomechanical simulation modeling and in surgery. When compared with joint function enabled by the current surgical practice of using sutures to make the attachment, biomechanical simulations show that the surgery with 1) the differential mechanism (tendon network) implant improves the fingers' ability to passively adapt to an object's shape significantly during grasping tasks $(2.74 \times$ as measured by the extent of finger flexion) for the same muscle force, and 2) the force-scaling implant increases knee-joint torque by $\mathbf{8 4 \%}$ for the same muscle force. The critical significance of this study is to provide a methodology for the design and inclusion of the implants into biomechanical models and validating the improvement in joint function they enable when compared with current surgical practice.
\end{abstract}

Index Terms-Biomechanical modeling, implant design, orthopedic surgery.

\section{INTRODUCTION}

B IOMECHANICAL simulation is a powerful tool for validating new surgical procedures and designs for new surgical implants. When compared with either in vivo or cadaver studies, biomechanical simulations enable quick and broad-based sensitivity analyses of how postsurgery

Manuscript received November 10, 2014; revised February 16, 2015; accepted March 21, 2015. Date of publication April 2, 2015; date of current version August 18, 2015. Asterisk indicates corresponding author.

${ }^{*}$ T. Homayouni is with the School of Mechanical, Industrial, and Manufacturing Engineering, Oregon State University, Corvallis, OR 97330 USA (e-mail: homayout@onid.oregonstate.edu).

K. N. Underwood and R. Balasubramanian are with the School of Mechanical, Industrial, and Manufacturing Engineering, Oregon State University.

K. C. Beyer and E. R. Martin are with the School of Chemical, Biological and Environmental Engineering, Oregon State University.

C. H. Allan is with School of Medicine, University of Washington.

This paper has supplemental material available online at http://ieeexplore. ieee.org (File size: $3 \mathrm{MB}$ ).

Color versions of one or more of the figures in this paper are available online at http://ieeexplore.ieee.org.

Digital Object Identifier 10.1109/TBME.2015.2419223



(a)



(b)
Fig. 1. Passive engineered mechanisms used as implants. (a) Using levers connect one muscle to four tendons in a hand. (b) Using a pulley between the quadriceps and the patella increases the knee joint torque. Legend: A-Vastus Medialis, B-Vastus Intermedius, C—Vastus Lateralis, D—Patella.

performance varies with changes to the surgical procedure and changes in the implant's design parameters, and how the new surgical procedure compares with prior art. This paper presents our study in the development of a novel class of biomechanical models for validating a new orthopedic surgical procedure that uses implants to improve the functional attachment between muscles and bone and tendons.

The new surgical procedure pioneered by our group uses implants in the form of passive engineered mechanisms for attaching muscles to bone or to one or more tendons with the goal of modifying the transmission of forces and movement within the body for improved musculoskeletal function (see Fig. 1 and [1]). The key motivation for this study is that the current method used primarily for attaching muscles to tendons and bone, namely the suture has drawbacks when used in orthopedic surgery, even though it has been the mainstay of surgery for 30000 years [2]. Specifically, the suture directly couples the movement of the muscle and the tendons/bone and provides limited ability to scale or distribute forces from the muscle across the tendons. This leads to limited postsurgery musculoskel-etal function and surgical choices. In contrast, the passive implants developed in this study can scale and/or distribute forces from the muscle across one or more tendons. This ability to modify the transmission of forces and movement between muscles and tendons and bones addresses the drawbacks that arise from the 
direct coupling that the suture creates in the surgery. In addition to improving joint function, it is expected that using the implants will provide increased options in orthopedic surgery.

To date, we have focused on two specific surgical applications: Application 1: Advancing tendon-transfer surgeries, where multiple tendons are attached to one muscle: the new surgical procedure uses a differential mechanism in the form of either pulleys, linkages, or tendon networks for distributing the forces from one muscle across multiple tendons to improve hand function in physical interaction tasks, such as the grasping of objects. Application 2: Advancing knee-replacement surgery: the new surgical procedure uses passive implants to scale the force created by the quadriceps muscles in order to increase knee-joint torque and, thus, improve joint performance in everyday tasks, such as stair climbing and chair rising.

While we have conducted pilot cadaver studies for Application 1 that demonstrate the feasibility of using one embodiment of a differential mechanism (namely a hierarchical pulley mechanism) [3], this paper presents how the implants are modeled in a biomechanical simulation platform (in particular, the OpenSim platform [4], [5]) and how the improvement in postsurgery function provided by the implants may be quantified (when compared with using the suture in current surgical procedure) through biomechanical simulations. Section II presents a background on relevant biomedical devices, OpenSim, and other biomechanical simulation platforms, and the two surgeries that are the current focus of our implant development work. Section III presents the implant models and how they are incorporated into OpenSim's upper and lower extremity biomechanical models. Section IV presents how the improved musculoskeletal function enabled by the implants can be quantified and compared with the function enabled by the current surgical procedure. Section V provides a discussion of the results and the paper concludes in Section VI.

\section{BACKGROUND}

\section{A. Biomedical Devices}

Prior work has explored three primary types of medical devices for restoring or enhancing musculoskeletal function: 1) rigid passive implants that directly attach to bones, such as joint-replacement implants [6] or implants for holding bones together after fractures [7]; 2) implants that secure two biological tendons, such as tenofix and ortho-Hunter implants [8], or biological tendons to bone, such as the orthocoupler implant [9]; 3 ) external devices that are body powered or externally powered, such as prostheses for replacing lost body parts, orthoses for correcting misalignments [10], or exoskeletons for assisting in movement [11]. Recently, there have been devices for directly attaching muscles in a residual limb to external prostheses through surgeries called cineplasty [12]. The proposed implants are differentiated from all existing medical devices since they modify force and movement transmission inside the body.

\section{B. Software Platforms for Biomechanical Modeling}

A variety of software platforms are available for modeling the biomechanics of the human musculoskeletal system and human movement, such as SIMM [13], Anybody [14], and OpenSim



(a)

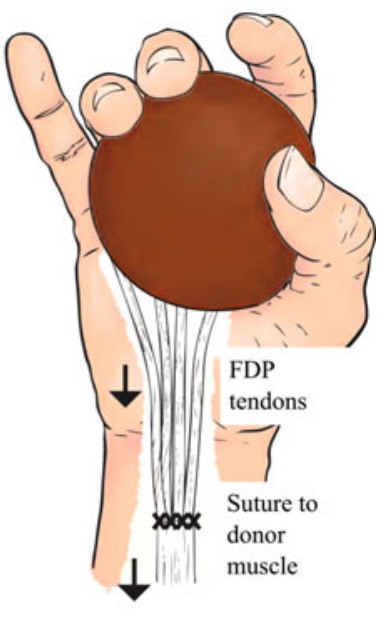

(b)
Fig. 2. Current tendon-transfer surgery. (a) Hand musculature. (b) Suturebased procedure.

[4], [5]. The basic components in each of these platforms are definitions of the bone geometry and joint kinematics [14], muscle force production models, and a dynamics engine that computes how the forces are transmitted through the skeletal kinematics [15]. We have chosen to use the OpenSim platform for our study since OpenSim is free and open source. Thus, it enables users to customize the upper and lower extremity models to be compatible with each patient anatomical variation [4].

The OpenSim program has been utilized extensively to model upper extremity and lower extremity human movements [5], [16] and how they are affected by different medical conditions and surgery. For example, OpenSim's hand models were used to explore improved routing of tendons for carpal tunnel syndrome and tendon disorders [17]. OpenSim's leg models were utilized to study the pre/postoperative balance associated with quadriceps insertion sites for patients with stiff knee gait [18]. However, all of these models only utilize natural elements such as bones and tendons or replace bone joints with rigid artificial elements. In contrast, the biomechanical models presented in this paper are unique from all previous work in that the models use reconfigurable mechanisms as implants that change the transmission of forces and movements between muscles and tendons or joints within the body.

\section{Application 1: Modeling of the Tendon-Transfer Surgery for Median-Ulnar Nerve Palsy}

Tendon-transfer surgeries are used to treat a variety of upper extremity conditions, such as stroke, palsy, spinal muscle atrophy, brain, nerve or muscle trauma, and congenital defects [19]. Each of these conditions severely affects hand function, and prevents a person from performing even basic activities of daily life. Tendon-transfer surgery restores lost hand function by rerouting one or more tendons from the affected muscle and suturing them to a functioning muscle (see Fig. 2). About 20000 hand tendon-transfer surgeries are performed annually in the U.S. alone at an estimated cost of \$200 million [20]. 
Our group has focused on developing implants for the tendontransfer surgery for high median-ulnar palsy, a severe condition that disables all four flexor digitorum profundus (FDP) muscle bellies. This condition results in an inability to fully close the fingers, leading to weak grasps [21]. The current surgical procedure addresses this condition by suturing all four FDP tendons to a functioning muscle, such as the extensor carpi radialis longus (ECRL). The ECRL muscle, which is a wrist extensor, has only one muscle belly. Thus, when the ECRL contracts, all the fingers curl inward simultaneously and equally.

The key problem with the current surgical procedure is that the suture couples the movement of all four fingers, leading to poor hand function in fundamental tasks such as grasping of objects since the fingers cannot adapt naturally to the object shape [19]. Specifically, the coupled finger movement leads to 1) incomplete and weak grasps [19], 2) greater forces being required of the muscle since the muscle has to isometrically stretch tendons to flex the other fingers once one finger makes contact (see Section IV-A Preliminary Results), 3) an uneven stretching of the tendons, which results in even more unbalanced finger movement over time [22], and 4) large unbalanced forces on the object during the grasping process (observed in robotic hands [23]). Also, significant challenges arise in current tendontransfer surgery due to the uncertainty in the surgical process [22], differences in biomechanical structure between patients, and the tradeoffs the surgeon must make when choosing from a limited set of donor muscles [24]. Specifically, if the surgeon makes even $5 \%$ error in tensioning the tendons, finger movement would be either premature or delayed during the grasping process, leading to weak grasps [1], [22]. Variation in the tendons' moment arms (the mechanical advantage the tendon has over a joint) and small deviations of the hand from the target object's center also leads to similar outcomes. Finally, to date, there is a little quantitative evaluation of how choices made in a tendon-transfer surgery affect hand function in physical interaction tasks such as grasping. Prior work only qualitatively evaluates a postsurgery hand function [25] or quantitatively evaluates finger and wrist movement in free space without external contact [26].

The current version of this tendon-transfer surgery has been modeled using OpenSim's upper extremity model [26]. We show in Section III how to model the modified surgery that uses implants.

\section{Application 2: Modeling of Total Knee-Replacement Surgery}

Knee-replacement surgery is performed in nearly 600000 patients a year in the U.S. at a total cost of $\$ 300$ billion [27]. In this surgery, the knee joint is replaced with a single degree-of-freedom artificial joint that mimics the knee's kinematics [28]. However, knee joint strength typically decreases by $30 \%$ following surgery for a variety of reasons, such as soft tissue disruption, implant pitting and fatigue, disturbance of the quadriceps mechanism (moment arms), tibiofemoral capsular dislocation, even though range of motion is unaffected [29]-[31]. Such joint-strength loss impedes daily activities, such

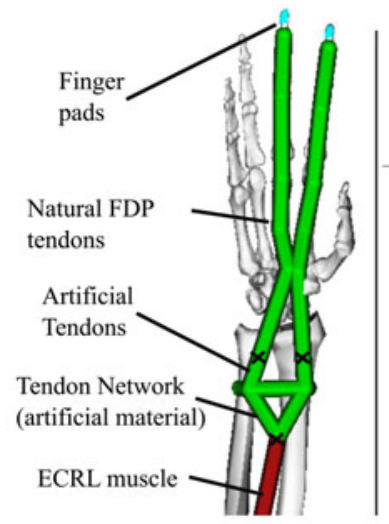

(a)

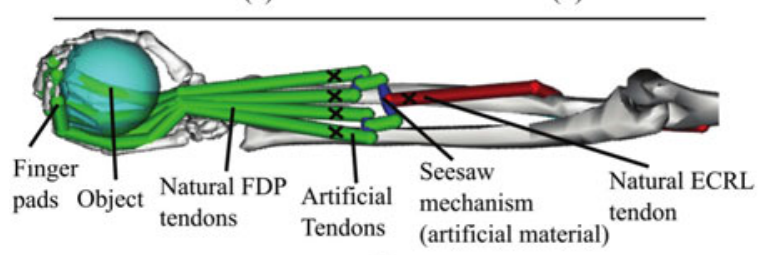

(c)

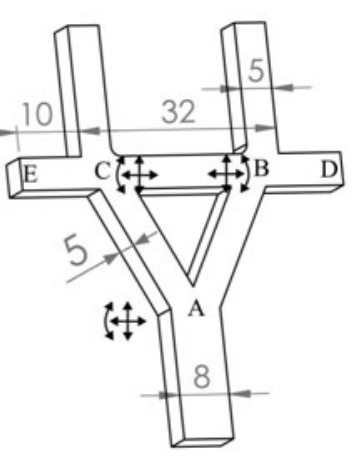

(b)
Fig. 3. Different hand differential mechanism simulated in OpenSim. (a) Tendon network. (b) Tendon network schematic, A, B, C are vertices of the network and each have three degrees of freedom each, D and $\mathrm{E}$ are anchor points. All sizes are in mm. (c) Seesaw mechanism.

as chair rising and stair climbing. Similar models have been used to predict the knee-load forces and joint torques using inverse kinematic [5].

\section{METHODS}

\section{A. Design of a Passive Implant to Create Differential Movement Between One Muscle and Multiple Tendons in Tendon-Transfer Surgery (Application 1)}

In order to address the problem of coupled movement that arises from the current tendon-transfer surgery for high medianulnar nerve palsy (see Section II-B), we have explored the use of a passive implant in the form of a differential mechanism to distribute the forces from one muscle to multiple tendons. The key idea is that a differential mechanism can route the forces and motion from a single muscle across multiple tendons, while allowing variable excursions in each tendon depending on the external contact the fingers make. The additional degrees of freedom provided by the implant to the muscle-tendon-finger biomechanics enable the fingers that have not made contact with the object to close even if other fingers have made contact ${ }^{1}$ [1]. We have designed two embodiments of the differential mechanism: 1) a tendon network [see Fig. 3(a) and (b)]; 2) a moving lever mechanism [see Fig. 3(c)]. A key challenge in the design of these mechanisms is that they should be small enough to fit inside the forearm, be able to withstand the physical stresses during operation, and be biocompatible. In this paper, we describe primarily how we address the design challenges of size

\footnotetext{
${ }^{1}$ This is similar to the use of a differential shaft in an automobile, which allows the four wheels to spin at different rates when negotiating a turn even though all the wheels are driven by a single engine.
} 
and biomechanical function. The issues of biocompatibility and material properties of the mechanism are beyond the scope of this paper. However, they are briefly described in Section V.

The tendon-network implant was added to the OpenSim upper extremity model using a web of tendons similar in properties to biological tendon (see Fig. 3). The tendon network has an equilateral triangular structure in order to distribute the forces and movement from one input (a muscle) across two outputs equally. This equilateral triangle is $32-\mathrm{mm}$ long on each side. The proximal end of the network is attached to the ECRL muscle, while the distal ends are attached to the finger tendons. The artificial tendons are chosen to have the same stiffness properties as biological tendons (normalized resistance force $F=1.6 x^{2}-1.4 x-0.2$, where $x$ is the tendon strain [4]); the physical version of the tendon network will be constructed from Kevlar [32] or polyvinylidene fluoride [33], which have favorable biological compatibility and mechanical properties [32]-[34]. Note that since the tendon network is not rigid, it is anchored to the forearm bones (the ulna and radius bones) in order to maintain its structure. The three ends of triangle [see A, B, C in Fig. 3(a)] are allowed to slide in a plane parallel to the bones and thus have three degrees of freedom each. The points $\mathrm{D}$ and E, however, are anchored to the bones. The same triangular structure can be hierarchically assembled to create differential action from one muscle across more than two tendons.

The moving-lever mechanism was added to the basic OpenSim upper extremity model in the form of a single rigid cylindrical element (these models may be designed in a 3-D modeling software, such as AutoCAD or created inside OpenSim itself). The ECRL muscle was connected at the center of the cylinder, and the FDP tendons were attached at the two ends of the cylinder [see Fig. 3(c)]. Note that offsetting the position of the ECRL attachment to either side of the center would create larger forces on the tendon on that side; thus, enabling the scaled distribution of forces between the fingers. The cylinder was provided three degrees of freedom to translate and rotate in the plane. One challenge with the lever mechanism is that it would have to be long for large muscle contractions. This is because the lever mechanism ceases to create differential action when it rotates beyond $80^{\circ}$. Specifically, our calculations show that the tendon of one finger could travel up to $2.5 \mathrm{~cm}$ with a $3 \mathrm{~cm}$ long lever after another finger is stopped due to contact during grasping. Since the ECRL's maximum excursion capability is $3.9 \mathrm{~cm}$, the patient would have to sacrifice some finger flexion capability for having the ability to scale the force transferred to the different fingers.

In all three models (model with the suture and models with the two implants), finger movement was created by setting the ECRL to have a linear-ramp-and-plateau activation profile (linear ramp over $3 \mathrm{~s}$ from $0 \%$ to $75 \%$ excitation, and then held at $75 \%$ for $3 \mathrm{~s}$ ). Contact between the fingers and the target object during the grasping process was simulated by creating contact between the index fingertip and an external rigid constraint as soon as the index finger flexed. The fingertips were set to be stiff with static, dynamic and viscous friction coefficients of $0.8,0.75$, and 0.01 in order to represent the physical interaction between the human fingertip and the objects for grasping.

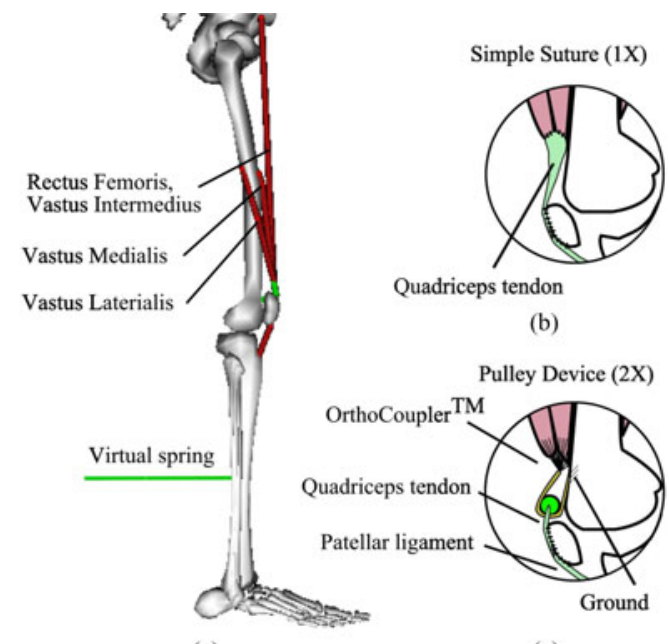

(a)

(c)

Fig. 4. Passive implant for scaling of muscle forces to improve knee function. (a) Implant simulation in OpenSim. (b) Knee musculature. (c) Knee musculature with the implant.

Finger movement was measured using the "total flexion" of all three flexion joints (MCP, PIP, and DIP) in the finger. The differential movement and fingertip force created by the muscle action across the index and middle fingers were measured in each simulation.

\section{B. Design of a Passive Implant for Scaling of Muscle Forces to Improve Knee Function Following Knee-Replacement Surgery (Application 2)}

In order to restore knee strength following knee-replacement surgery, we propose implanting a passive pulley mechanism between the quadriceps and the patella. The pulley would scale the quadriceps force by about $200 \%$, while sacrificing about $50 \%$ of the range of motion. Such partial loss of range of motion in the knee is acceptable, because only $105^{\circ}$ of movement is necessary for daily activities. The pulley was incorporated into the basic OpenSim lower extremity model by inserting and attaching an additional tendon to the four quadriceps muscle heads, routing it through a via point on the patella, and then anchored to the femur bone. This routing acts as a pulley because the tendon is free to slide around the via point on the patella [see Fig. 4(c)]. In both models with and without the implant, knee joint movement was created by setting all four quadriceps muscles to have a linear-ramp-and-plateau activation profile (linear ramp over $3 \mathrm{~s}$ from $0 \%$ to $75 \%$ excitation, and then held at $75 \%$ for $3 \mathrm{~s})$. The knee joint torque was estimated using the force in a virtual spring attached to the tibia. This spring was modeled in OpenSim using a tendon with the same force-length curve as biological tendons. The knee joint's initial position was $54^{\circ}$, and the knee joint's change in angle was measured.

\section{Key Challenges in Incorporating Implants Into Biomechanical Models}

There were two challenges that needed to be overcome when incorporating these mechanisms into the biomechanical models. 




Fig. 5. Middle finger flexion enabled by the implant-based procedure and the suture-based procedure.

First, the tendon lengths needed to be chosen carefully so the biomechanical function is not affected. Specifically, if the tendons were too slack, then the transfer of the muscle contraction to the joint would be delayed. If tendons were too tight, then that would lead to premature movement in the joints. Second, the implant can also change where along muscle force-length curve the muscle operates [35]. Therefore, effort was taken to ensure that the muscle operated in the same section of the force-length curve with and without the implant.

\section{RESUlTS}

\section{A. Improved Differential Finger Movement Following Implant-Based Surgical Procedure (Application 1)}

Fig. 5 shows the large difference in finger flexion enabled by the implanted tendon-network and moving-lever mechanisms when compared with the finger flexion enabled by the suturebased procedure when the fingers close in to grasp an object of uneven shape. Specifically, both the implanted tendon-network and moving-lever mechanisms provide nearly $170^{\circ}$ of differential movement between the index and middle fingers, which is nearly three times the differential movement $\left(62^{\circ}\right)$ enabled by the suture-based procedure. It was confirmed that the ECRL force was equal in all three cases, $275(\mathrm{~N})$, confirming that the differential action was due to the implanted mechanism. Conversely, it was also observed that less muscle force was required to close the fingers to the same extent following the implantbased procedure. Thus, when establishing a multifinger power grasp, the fingers would adapt better to the object's shape at lower actuation force following the implant-based procedure.

While the above results show that the implant-based procedure improves finger adaptability during power grasps, the influence of the suture-based and implant-based procedures during index-thumb precision grasp [36] was also analyzed. Specifically, the suture-based procedure enables the immediate creation of a precision grasp once the index finger makes contact with the object, since the movement of all the fingers ceases immediately after the index finger makes contact. Thus, the contact force established between the index finger and the object is expected to be large. In the case of the implant-based procedure, strong contact forces can be established only after the other

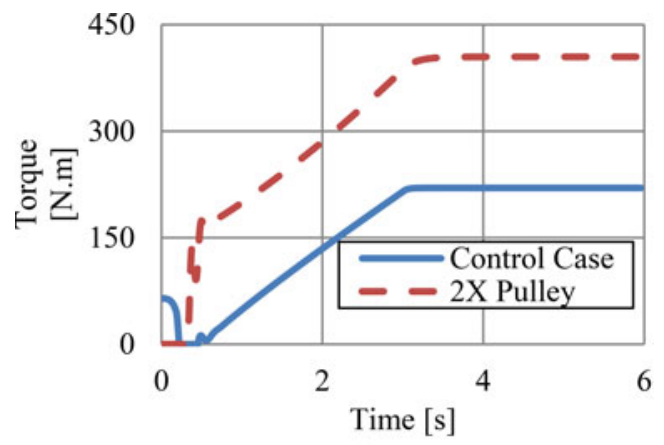

(a)

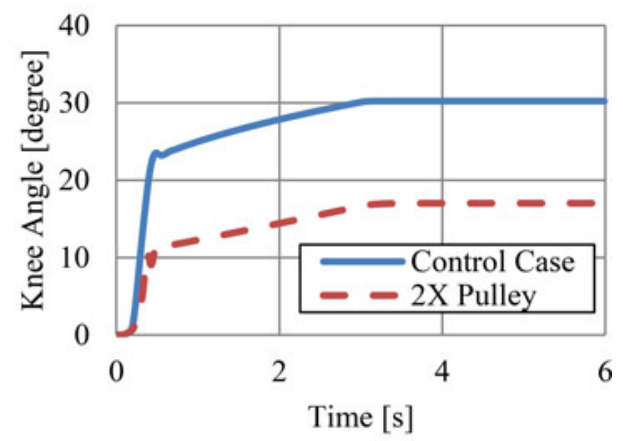

(b)

Fig. 6. (a) Knee joint force and (b) movement with and without the implant.

fingers also close in. This marginally reduces the contact forces that may be established in the precision grasp following the implant-based surgery. However, the simulations showed that the fingertip force enabled by the implant-based procedure in pinch grasps is still comparable to the fingertip force of a healthy person (about 27.9 N) [37]. Thus, the implant-based procedure is able to create differential action between multiple fingers to improve power grasping capability, while also enabling sufficient strength in pinch grasps similar to that of a healthy individual.

\section{B. Increased Knee-Joint Torque Following Implant-Based Surgical Procedure (Application 2)}

Fig. 6(a) and (b) shows the time history of the knee-joint torque and the knee-joint angle change due to quadriceps activation following the standard surgery and following the implantbased surgery. It is observed that the knee-joint torque generated following the implant-based surgery is $1.84 \mathrm{X}$ the force generated in the suture-based procedure. A force scaling of $2 \mathrm{X}$ was expected, but the reduced scaling arises from the irregular lines of action for muscles and ligaments in the human body when compared with mechanical systems [38]. Specifically, if the directions of the input and output tendons are not perfectly aligned or in the plane, the angular discrepancy will affect the magnitude of force scaling. The range of motion correspondingly decreased $0.56 \mathrm{X}$ when using the implant.

\section{DISCUSSION}

The long-term goal of this study is to develop implants that provide an alternative to the suture (or other methods like the surgical staple [39]) for attaching muscles to tendons and bones 


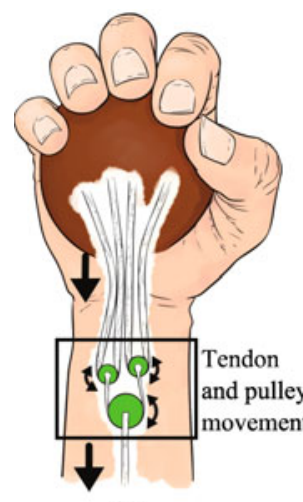

(a)



(b)
Fig. 7. Application 1, differential movement. (a) Pulley configuration. (b) Testing pulley implant in cadaver.

in surgery. Such implants have the potential to bring a paradigm shift in reconstructive orthopedic surgery since these implants can customize the transmission of forces and movement within the body based on the patient's requirements, which has never been explored in surgery. It is expected that these implants will help restore musculoskeletal function and provide more options in surgery.

The implants have to be carefully designed keeping in mind that biomechanical function is highly sensitive to small alterations in muscle and tendon lengths and routings and where the muscle operates in the force-length curve [35]. For example, it was noticed during the design process for the knee implant that any additional tendon length would result in the muscle operating at lengths where the force output is not maximum. Also, the implants can be customized based on the musculoskeletal function the patient desires. Specifically, by varying the stiffness and dimensions of various elements of the tendon network in Application 1, the movement of the index and middle fingers can be selectively modified to achieve greater index finger movement (when compared with the middle finger movement). This would enable the preferential creation of index-thumb precision grasps over enveloping power grasps. This will be investigated in future work.

Finally, we have successfully demonstrated the performance of these implants in cadavers in the case of application 1, namely tendon-transfer surgery for high median-ulnar nerve palsy using the pulley system (see Fig. 7) [1], [3]. However, it is logistically difficult and expensive to do extensive studies and optimize different embodiments of the implants using cadavers. Thus, biomechanical simulations provide us an important route for evaluating various embodiments of the implants.

\section{CONCLUSION}

The key contribution of this paper is to provide the methodology for incorporating and validating these mechanisms in biomechanical simulation programs such as OpenSim. The beneficial impact of these implants on biomechanical function is evident from the results of the OpenSim simulations. Specifically, in Application 1, the implanted differential mechanism enables the fingers to adapt better to the object shape during grasping tasks. In Application 2, the pulley mechanism enables significantly greater knee joint torque for the same muscle activation (see Figs. 5 and 6).

While these results are promising, additional experiments and analysis are necessary in order to verify how the implants will perform when embedded amidst living tissue. First, it is not yet clear if the implant will damage surrounding tissue when the passive mechanism moves and rotates in vivo due to muscle contraction [40]. Such physical interaction between the implant and surrounding tissue may be quantified through wetlab experiments and software, such as ANSYS that enables a mechanobiology analysis [41]. Second, finite-element analysis is necessary to analyze how the implant should be dimensioned in order to withstand the forces that the muscle creates in the implant. Third, in addition to fabricating the device using biocompatible materials such as titanium or ultrahigh molecular weight polyethylene, the mechanism may have to be chemically coated to reduce fibrosis when implanted in vivo long-term [42].

\section{REFERENCES}

[1] R. Balasubramanian et al., "Implanted miniature engineering mechanisms in tendon-transfer surgery improve robustness of post-surgery hand function," in Proc. 6th Hamlyn Symp. Med. Robot., 2013, pp. 29-30.

[2] D. Mackenzie, "The history of sutures," Med. History, vol. 17, no. 2, pp. 158-168, 1973.

[3] K. L. Mardula et al., "Implanted passive engineering mechanism improves hand function after tendon transfer surgery: A cadaver-based study," HAND, vol. 10, pp. 116-122, Sep. 2014.

[4] S. L. Delp et al., "OpenSim: Open-source software to create and analyze dynamic simulations of movement," IEEE Trans. Biomed. Eng., vol. 54 no. 11, pp. 1940-1950, Nov. 2007.

[5] E. M. Arnold et al., "A model of the lower limb for analysis of human movement," Ann. Biomed. Eng., vol. 38, no. 2, pp. 269-279, 2010.

[6] U. Wijk et al., "Outcomes of proximal interphalangeal joint pyrocarbon implants," J. Hand Surg. Amer., vol. 35, no. 1, pp. 38-43, 2010.

[7] I. J. Harrington and A. A. Tountas, "Replacement of the radial head in the treatment of unstable elbow fractures," Injury, vol. 12, no. 5, pp. 405-412, 1981.

[8] B. W. Su et al., "A device for zone-II flexor tendon repair," J. Bone Joint Surg., vol. 88, no. 1 Suppl. 1, pp. 37-49, 2006.

[9] A. Melvin et al., "An artificial tendon to connect the quadriceps muscle to the Tibia," J. Orthop. Res., vol. 29, no. 11, pp. 1775-1782, 2011

[10] D. Shurr and J. Michael, Prosthetics and Orthotics. Englewood Cliffs, NJ, USA: Prentice-Hall, 2001.

[11] J. C. Perry et al., "Upper-limb powered exoskeleton design," IEEE/ASME Trans. Mechatronics, vol. 12, no. 4, pp. 408-417, Aug. 2007.

[12] R. F. Weir et al., "Cineplasty as a control input for externally powered prosthetic components," J. Rehabil. Res. Dev., vol. 38, no. 4, pp. 357-63, Jan. 2001.

[13] SIMM (Software for Interactive Musculoskeletal Modeling), Musculographics, Inc., A division of motion analysis corporation, Santa Rosa, CA, USA.

[14] M. Damsgaard et al., "Analysis of musculoskeletal systems in the AnyBody modeling system," Simul. Model. Pract. Theory, vol. 14, no. 8, pp. 1100-1111, Nov. 2006.

[15] R. Shadmehr and S. P. Wise, The Computational Neurobiology of Reaching and Pointing: A Foundation for Motor Learning (Computational Neuroscience Series). Cambridge, MA, USA: MIT Press, 2005.

[16] S. R. Ward et al., "Are current measurements of lower extremity muscle architecture accurate?" Clin. Orthop. Relat. Res., vol. 467, no. 4, pp. 1074-1082, 2009.

[17] A. M. Kociolek and P. J. Keir, "Modelling tendon excursions and moment arms of the finger flexors: Anatomic fidelity versus function," J. Biomech., vol. 44, no. 10, pp. 1967-73, Jul. 2011.

[18] D. H. Sutherland et al., "Treatment of stiff-knee gait in cerebral palsy: A comparison by gait analysis of distal rectus Femoris transfer versus proximal rectus release," J. Pediatr. Orthop., vol. 10, no. 4, pp. 433-441, 1990. 
[19] P. W. Brand and A. Hollister, Clinical Mechanics of the Hand. St. Louis, MO, USA: Mosby, 1993.

[20] AHA-UW. (2013). Based on American Hospital Association and University of Washington Medicine records. [Online]. Available: http://www.aha.org/research/rc/stat-studies/fast-facts.shtml

[21] D. M. Sammer and K. C. Chung, "Tendon transfers: Part I. Principles of transfer and transfers for radial nerve palsy," Plastic Reconstr. Surg., vol. 123 , no. 5, pp. 169e-177e, May 2009.

[22] R. L. Lieber, "Biology and mechanics of skeletal muscle: What hand surgeons need to know when tensioning a tendon transfer," J. Hand Surg. Amer., vol. 33, no. 9, pp. 1655-1656, 2008.

[23] A. M. Dollar and R. D. Howe, "The highly adaptive SDM hand: Design and performance evaluation," Int. J. Robot. Res., vol. 29, no. 5, pp. 585-597, 2010.

[24] J. Fridén and R. L. Lieber, "Tendon transfer surgery: Clinical implications of experimental studies," Clin. Orthop. Relat. Res., vol. 403, pp. S163S170, 2002.

[25] D. E. Beaton et al., "The DASH (disabilities of the arm, shoulder and hand) outcome measure: What do we know about it now?" Brit. J. Hand Ther, vol. 6, no. 4, pp. 109-118, 2001.

[26] K. R. S. Holzbaur et al., "A model of the upper extremity for simulating musculoskeletal surgery and analyzing neuromuscular control," Ann. Biomed. Eng., vol. 33, no. 6, pp. 829-840, Jun. 2005.

[27] A. J. Carr et al., "Knee replacement," Lancet, vol. 379, no. 9823, pp. 1331-1340, 2012.

[28] R. L. Kane et al., Total knee replacement, US Department of Health and Human Services, Public Health Service, Agency for Healthcare Research and Quality, Washington, DC, USA, 2003.

[29] (2011, Oct. 22). Tendon Transfer Principles and Mechanics. [Online]. Available: http://emedicine.medscape.com/ article/1834677-overview

[30] B. T. Rougraff et al., "Complete quadriceps tendon ruptures," Orthopedics, vol. 19 , no. 6, pp. 509-514, 1996

[31] J. G. Kim et al., "The effective-ness of minimally invasive total knee arthroplasty to preserve quadriceps strength: A randomized controlled trial," Knee, vol. 18, no. 6, pp. 443-447, Dec. 2011.

[32] B. Pourdeyhimi et al., "Fracture toughness of Kevlar 29/poly (methyl methacrylate) composite materials for surgical implantations," Ann. Biomed. Eng., vol. 14, no. 3, pp. 277-294, May 1986.
[33] G. Laroche et al., "Polyvinylidene fluoride (PVDF) as a biomaterial: From polymeric raw material to monofilament vascular suture," J. Biomed. Mater. Res., vol. 29, no. 12, pp. 1525-36, Dec. 1995.

[34] J. H.-C. Wang, "Mechanobiology of tendon," J. Biomech., vol. 39, no. 9, pp. 1563-1582, 2006.

[35] C. N. Maganaris, "Force-length characteristics of in vivo human skeletal muscle," Acta Physiol. Scand., vol. 172, no. 4, pp. 279-285, 2001.

[36] J. H. House et al., "Restoration of strong grasp and lateral pinch in tetraplegia due to cervical spinal cord injury," J. Hand Surg. Amer, vol. 1, no. 2, pp. 152-159, Sep. 1976.

[37] F. J. Valero-Cuevas et al., "Large index-fingertip forces are produced by subject-independent patterns of muscle excitation," J. Biomech., vol. 31, no. 8, pp. 693-703, Aug. 1998.

[38] A. V. Specogna et al., "Radiographic measures of knee alignment in patients with varus gonarthrosis: Effect of weightbearing status and associations with dynamic joint load," Amer. J. Sports Med., vol. 35, no. 1, pp. 65-70, Jan. 2007.

[39] J. D. Bronzino, The Biomedical Engineering Handbook. 2. Boca Raton, FL, USA: CRC Press, 2000.

[40] D. R. Carter et al., "Correlations between mechanical stress history and tissue differentiation in initial fracture healing," J. Orthop. Res., vol. 6 , no. 5, pp. 736-48, Jan. 1988.

[41] C. Zhao et al., "Soft tissue deformation ANSYS simulation of robotassisted percutaneous surgery," in Proc. 10th World Congr. Intell. Control Autom., 2012, pp. 3561-3566.

[42] L. Zhang et al., "Zwitterionic hydrogels implanted in mice resist the foreign-body reaction," Nature Biotechnol., vol. 31, no. 6, pp. 553-556, 2013.

Authors' photographs and biographies not available at the time of publication. 